[Article]

\title{
激光诱导铝纳米膜熔化机理的分子动力学模拟
}

\author{
白明泽 ${ }^{1}$ 程 丽 $^{1}$ 唐 红 ${ }^{1}$ 豆育升 ${ }^{1,2, *}$
}

('重庆邮电大学计算化学研究所, 重庆 400065; ${ }^{2}$ Department of Physical Sciences, Nicholls State University, LA 70310, USA)

\begin{abstract}
摘要: 采用耦合一维双温模型的分子动力学方法研究了纳米级的铝膜在飞秒激光辐照下的熔化机制. 这种方 法不仅能够在原子水平上展现金属膜的各种微观行为, 还能有效地描述金属膜的激光能量吸收、传递和金属电 子热传导等过程. 模拟结果表明, 与其它金属相比, 铝膜在飞秒激光辐照下的电子温度、晶格温度以及内部压力 等呈现出不同的变化. 铝膜在较高强度激光辐照下会很快发生全局一致的熔化, 这与镍膜上下非均匀的熔化不 同. 并且由于铝的电子-声子耦合强度较高导致铝膜较镍膜和金膜熔化得更快. 模拟结果显示, 铝膜的熔化时间 与实验测量的超快激光诱导的铝膜熔化时间一致. 进而从理论上支持激光诱导的铝膜熔化是一个热力学熔化 过程.
\end{abstract}

关键词: 金属铝; 激光熔化; 双温模型; 超快动力学; 热力学熔化

中图分类号: $0641 ; 0643$

\section{Molecular Dynamics Simulation of the Laser-Induced Melting of an Al Nanofilm}

\author{
BAI Ming-Ze ${ }^{1}$ CHENG Li $i^{1} \quad$ TANG Hong ${ }^{1} \quad$ DOU Yu-Sheng ${ }^{1,2, *}$ \\ ('Institue of Computational Chemistry, Chongqing University of Posts and Telecommunications, Chongqing 400065, P. R. China; \\ ${ }^{2}$ Department of Physical Sciences, Nicholls State University, LA 70310, USA)
}

\begin{abstract}
A coupled computational technique, which combines the one dimensional two-temperature model and molecular dynamics, was used to study the melting dynamics of a nanoscale aluminum film irradiated by a femtosecond laser pulse. The model is capable of providing an atomic-level depiction of fast microscale processes in metals and gives an adequate description of laser light absorption, energy transfer, and fast electron heat conduction in metals. The simulation revealed that the electron temperature, lattice temperature, and laser induced pressure of the Al film were significantly different from those of $\mathrm{Ni}$ and $\mathrm{Au}$ films. The Al film melts globally soon after laser radiation and this is different from the Ni film, which goes through a step melting process. In addition, the Al film shows a much faster melting process than the $\mathrm{Ni}$ and $\mathrm{Au}$ films because of strong electron-phonon coupling. The melting time of the Al film by an ultrafast laser pulse is consistent with recent experimental observations, which supports the assertion that the laser induced melting of an $\mathrm{Al}$ film is a thermal process.
\end{abstract}

Key Words: $\quad$ Metal Al; Laser melting; Two-temperature model; Ultrafast dynamics;

Thermal melting

Received: July 22, 2010; Revised: October 12, 2010; Published on Web: November 10, 2010.

*Corresponding author. Email: Yusheng.Dou@nicholls.edu; Tel: +86-23-62461438.

The project was supported by the National Natural Science Foundation of China $(20773618,21073242)$ and Natural Science Foundation of Chongqing University of Posts and Telecommunications, China (A2008-39).

国家自然科学基金(20773618,21073242)和重庆邮电大学自然科学基金(A2008-39)资助项目

(C) Editorial office of Acta Physico-Chimica Sinica 
激光辐照金属技术已被广泛地应用于工业领 域, 如激光切割/钻孔、激光焊接、激光打标等 ${ }^{[1-4]}$. 但 是激光与金属的相互作用机理还没有得到充分的 理解. 铝及其合金具有耐蚀性, 导电性、导热性好和 比强度高等特点, 在工业领域获得广泛应用, 其中 很多都涉及铝合金的激光熔解过程 ${ }^{[5-6]}$. 因此, 研究 铝和激光的相互作用显得尤为重要. 目前激光作用 下铝的熔化机理仍是一个有争论的课题. Guo 等 ${ }^{[7]}$ 用 光百浦-探测检测介电常数的方法研究了受超强激 光辐照时多晶铝内部结构的变化. 他们发现铝在激 光辐照后 $500 \mathrm{fs}$ 就呈现出液相, 这个时间远小于晶 格受热所需时间, 因此他们认为激光导致的铝熔化 是一个非热力学过程.

后来 Kandyla 等 ${ }^{[8]}$ 采用最新发展的光洜浦-探测 方法再次检测铝的熔化过程, 与 Guo 等人的实验技 术相比, Kandyla等采用的方法具有宽频带和高时 间分辨率等特点. 该测量技术能检测金属熔化过程 中较宽频带范围(1.7-3.5 eV)的光学性质, 时间分辨 率为 $65 \mathrm{fs}$. 他们的研究发现在各种强度激光辐照下 铝膜的熔化出现在 1.5-2.0 ps 时间内. 根据这一研 究结果, 高强度激光辐照下铝膜的熔化是一个热力 学过程.

最近发展的超快电子衍射方法可以检测出金 属内部原子的结构变化. Siwick 等 ${ }^{[9]}$ 采用时间分辨 率为 $500 \mathrm{fs}$ 的超快电子衍射探测方法发现铝在飞秒 激光辐照下, 需要 $3.5 \mathrm{ps}$ 的时间来完成熔化. 为了解 释实验结果, 他们提出铝的熔化是由于晶格快速过 热而产生的晶格崩塌而不是非热熔化: 在激光辐照 作用下, 热电子会在几十飞秒内从初始的带间跃迁 快速分布而形成导带内的热电子分布 ${ }^{[10]}$. 这种激光 引起的快速的电子再分布与半导体内导带上持续 时间相对较长的电子分布有很大的不同, 这些金属 内的电子会快速地弛豫到初始电子能带上, 因而没 有足够的空间微扰来实现非热力学熔化过程. 然后 高温的热电子会通过电子-声子耦合来加热晶格. 超 快电子衍射实验的缺点是仪器的时间分辨率低, 对 于研究激光诱导铝熔化这种介于热力学和非热力 学之间的过程会遇到困难. 并且 Siwick 等人对于他 们实验结果的解释缺乏理论上的支持.

与实验方法相辅助的研究方法是分子动力学 (MD)模拟方法 ${ }^{[11-12]}$. MD 模拟在每一个时间步根据 原子坐标以及选用的力场计算每个原子应受的力, 然后根据牛顿第二定律计算原子的位移. 重复计算
多个时间步可获得体系中每个原子的精确运动轨 迹, 因而可在原子尺度描述材料内部的动力学行 为. 对于金属的许多性质, MD模拟可以取得与实验 数据相当一致的结果 ${ }^{[13-15]}$. 利用 MD 模拟研究金属 激光相互作用时的一个重要难题是如何正确处理 激光能量的吸收和传递. 传统的方法是将激光能量 通过调节原子速率的方法直接附加给幅射层内的 原子 ${ }^{[16]}$, 该方法的缺点是不能有效地描述金属激光 相互作用以及能量的传递过程.

Anisimov 等 ${ }^{[17]}$ 提出的双温模型(TTM) 能够在连 续水平上描述电子晶格温度变化的机理. 该模型的 基本思想是: 金属中的电子在极短的时间(飞秒级) 内吸收激光的光子能量, 使得电子温度快速上升. 而电子的能量向晶格传递需通过较慢的电子-晶格 耦合作用, 通常需要皮秒级的时间才能达到平衡. 平衡前的电子体系和晶格体系的温度差别很大, 形 成所谓的电子-晶格双温体系. 双温体系中电子和晶 格温度的变化受到电子热容、热传导、电子-声子耦 合强度、晶格热容热传导等性质的影响. Zhigilei 研 究组 ${ }^{[18]}$ 将这些对电子和晶格温度变化规律的描述用 于 $M D$ 模拟方法, 从而发展了耦合的双温-分子动力 学(TTM-MD)的模拟技术. 并应用该方法研究了金 和镍等金属在激光辐照下一系列性质的变化 ${ }^{[18-19]}$. 铝与镍及金在电子热容、热传导、电子-声子耦合强 度、晶格热容以及热传导等性质上有明显的差异, 因此预期在飞秒激光辐照下会表现出不同的反应.

本文采用耦合的 TTM-MD 模型模拟研究铝膜 在飞秒激光辐照下熔化的动力学过程, 从而在原子 水平上理解激光诱导铝熔化的机理.

\section{1 模拟方法}

当激光辐照金属时, 金属导带电子会吸收光子 能量, 吸收的能量迅速地在飞秒时间尺度内传导到 整个电子体系并达到平衡. 电子热能转换成原子振 动能的过程相对缓慢, 电子与声子之间的热平衡由 电子-声子耦合强度决定, 通常在皮秒级时间内达 到. 如果激光辐照的时间小于或等于电子-声子平衡 所需的时间, 体系就会进入非平衡态. 晶格温度 $T_{1}$ 和 电子温度 $T_{\mathrm{e}}$ 随时间的变化可以用一个连续的双温模 型 ${ }^{\left[{ }^{[17}\right]}$ 来描述. 双温模型包含下面两个非线性微分方 程:

$$
C_{\mathrm{e}}\left(T_{\mathrm{e}}\right) \frac{\partial T_{\mathrm{e}}}{\partial t}=\nabla\left[K_{\mathrm{e}}\left(T_{\mathrm{e}}\right) \nabla T_{\mathrm{e}}\right]-G\left(T_{\mathrm{e}}-T_{\mathrm{l}}\right)+S(z, t)
$$




$$
C_{1}\left(T_{1}\right) \frac{\partial T_{1}}{\partial t}=\nabla\left[K_{1}\left(T_{1}\right) \nabla T_{1}\right]+G\left(T_{\mathrm{e}}-T_{1}\right)
$$

其中 $C$ 和 $K$ 表示电子(下标 $\mathrm{e}$ )和晶格(下标 1 )的热容 和热传导系数. $G$ 是电子-声子耦合系数. $S(z, t)$ 描述 单位面积单位时间内吸收的激光能量. 用有限差分 法求解式(1)和(2)可以得到电子和晶格温度随时间 和空间的变化情况.

在耦合的 TTM-MD 模型中, 描述电子温度 $T_{\mathrm{e}}$ 扩 散情况的式(1)采用有限差分法求解, 而晶格温度的 计算以及电子晶格间的能量交换则采用分子动力 学方法 ${ }^{[18-19]}$. 在耦合模型中的 TTM 为一维模型时, 首先将模拟体系沿 $z$ 方向划分为有限个计算单元， 应用式(3)和(4)可以得到每个单元的电子和晶格温 度以及每个原子的运动轨迹,

$$
\begin{gathered}
\text { TTM: } C_{\mathrm{e}}\left(T_{\mathrm{e}}\right) \frac{\partial T_{\mathrm{e}}}{\partial t}=\frac{\partial}{\partial z}\left[K_{\mathrm{e}}\left(T_{\mathrm{e}}\right) \frac{\partial T_{\mathrm{e}}}{\partial z}\right]- \\
G\left(T_{\mathrm{e}}-T_{\mathrm{l}}\right)+S(z, t) \\
\text { MD: } m_{i} \frac{\mathrm{d}^{2} r_{i}}{\mathrm{~d} t^{2}}=F_{i}+\xi m_{i} v_{i}^{T} \\
\text { 其中 } \xi=\frac{1}{n} \sum_{k=1}^{n} G V_{N}\left(T_{\mathrm{e}}^{k}-T_{1}\right) / \sum_{i} m_{i}\left(v_{i}^{T}\right)^{2}
\end{gathered}
$$

式(4)中 $m_{i}$ 和 $r_{i}$ 分别是原子 $i$ 的质量与位置, $F_{i}$ 是所有 其它原子作用在原子 $i$ 上的力的和, $v_{i}^{T}$ 为原子 $i$ 的热 运动速度, $V_{N}$ 为单元格体积, $n$ 为 $\mathrm{MD}$ 积分步长与有 限差分法步长的商. 关于这些参数的详细解释参见 文献 ${ }^{[18]}$.

图 1 为描述耦合的 TTM-MD 模型的示意图, 每 个小格子代表了双温模型中电子温度和晶格温度 的计算单元, 格子中不同颜色的小球代表分别处于 液相和固相环境中的原子. 对于体系中的原子, 除 了通过传统的 MD力场根据周围原子坐标计算它受 的力外, 还要计算与电子向晶格传递的能量所对应 的力(见式(4)).

该模型结合了双温模型和 MD 方法各自的优 点. 双温模型可以较好地描述如下物理过程: 电子 体系对能量的吸收、电子与声子间能量的传递以及
电子在金属中的快速热传导等. MD 方法可计算出 每个原子与周围原子的相互作用力以及电子热传 递能量影响产生的运动轨迹, 从而能较精确地描述 金属膜受激光辐照过程中晶格过热、熔化、喷溅等 非平衡过程.

$\mathrm{MD}$ 模拟体系包含 69120 个 $\mathrm{Al}$ 原子, 产生的三 维体积为 $4.93 \mathrm{~nm} \times 4.93 \mathrm{~nm} \times 49.3 \mathrm{~nm}$. 在 $x$ 和 $y$ 方向 (垂直于激光入射方向)设置二维周期边界条件, 保 持 $z$ 方向(平行于激光入射方向)为自由边界.

铝膜的所有热力学和弹性性能, 如晶格热容、 弹性模量、热膨胀系数、熔化温度、熔化的体积以及 这些性能与温度和压力的依赖关系由嵌入原子势 $(\mathrm{EAM})^{[20-21]}$ 和 Johnson 等 ${ }^{[22]}$ 发展的铝的 EAM 参数来 计算. 铝的光穿透深度选择 $15 \mathrm{~nm}^{[23]}$. 式(3)中电子体 系所用到的铝材料参数选择如下: 电子热容 $C_{\mathrm{e}}=\gamma T_{\mathrm{e}}$, $\gamma=92 \mathrm{~J} \cdot \mathrm{m}^{-3} \cdot \mathrm{K}^{-2}$; 电子热传导系数 $K_{\mathrm{e}}=K_{0} T_{\mathrm{e}} / T_{1}, K_{0}=238$ $\mathrm{W} \cdot \mathrm{m}^{-1} \cdot \mathrm{K}^{-1}$; 电子-声子耦合系数 $G=4.9 \times 10^{17} \mathrm{~W} \cdot \mathrm{m}^{-3}$. $\mathrm{K}^{-1[24]}$

为了正确描述晶格的温度, 图 1 所示 $z$ 方向空间 步长 $\Delta \chi_{\mathrm{FD}}$ 被设为 $1 \mathrm{~nm}$. MD积分步长设为 $2 \mathrm{fs}$, 有限 差分步长为积分步长的 $1 / 50$, 即 $0.04 \mathrm{fs}$. 在弛豫过程 中体系总能量的波动局限在 $0.03 \%$ 以内, 证明前面 所选参数值是合理的. 本研究所采用的激光脉宽为 $200 \mathrm{fs}$, 吸收强度在 70-170 J $\cdot \mathrm{m}^{-2}$ 范围内. 在激光照 射之前铝膜体系已平衡到温度 $300 \mathrm{~K}$ 、压力 $0 \mathrm{GPa}$ 的 状态, 此时密度为 $2.59 \mathrm{mg} \cdot \mathrm{cm}^{-3}$.

\section{2 结果与讨论}

激光辐照金属时, 激光能量最开始由导带电子 吸收, 之后在电子体系内快速地进行热传导, 并逐 步通过电子-声子耦合将能量传递到晶格, 所吸收的 能量最终分布到以下几种能量形式中: 电子的热能 $E_{\mathrm{e}}$ 、与原子热运动相关的能量 (包括动能与势能) $E_{\mathrm{a}}^{\text {th }}$ 、 与原子集体运动有关的能量 $E_{\mathrm{a}}^{\mathrm{c}}$ 以及熔化潜热消耗 的能量 $E_{\mathrm{m}}$ 等. 其中 $E_{\mathrm{a}}^{\mathrm{th}}$ 与 $E_{\mathrm{a}}^{\mathrm{c}}$ 均含在体系的总动能和总

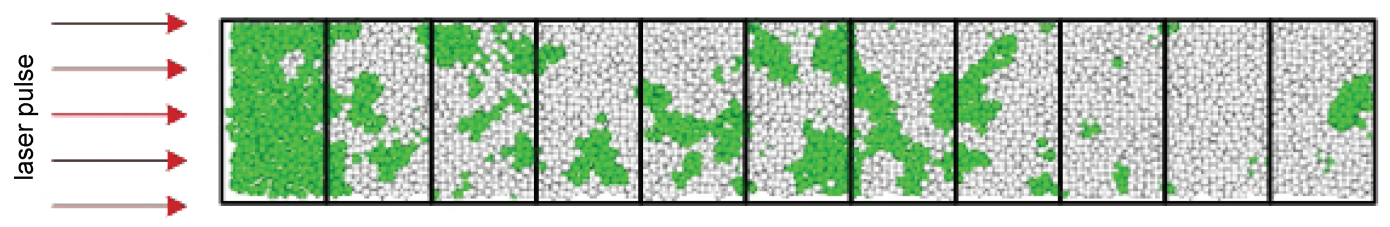

图 1 耦合的 TTM-MD 模型示意图

Fig.1 Schematic representation of the coulped TTM-MD model 

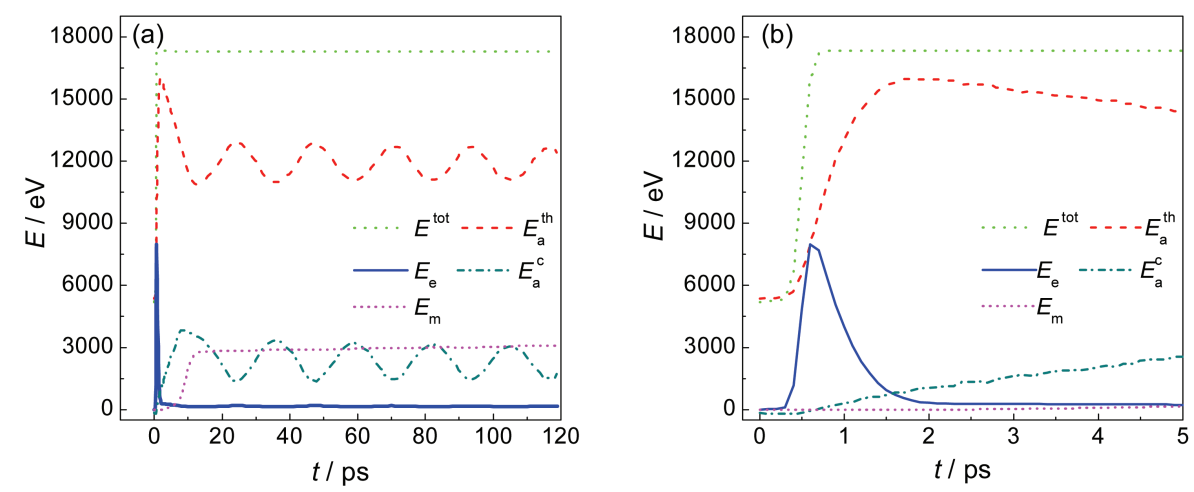

图 2 飞秒激光辐照铝膜后的能量分布图

Fig.2 Energy redistribution of the Al film irradiated with a femtosecond laser

Pulse width is $200 \mathrm{fs}$ and absorbed fluence is $77 \mathrm{~J} \cdot \mathrm{m}^{-2} ; E^{\text {tot }}$ : the total energy of the system, $E_{\mathrm{a}}^{\text {th }}\left(\right.$ or $E_{\mathrm{c}}$ ): the thermal energies of the atoms (or electrons), $E_{\mathrm{a}}^{\mathrm{c}}$ : the energy of the collective atomic motion, $E_{\mathrm{m}}$ : the energy going to the latent heat of melting

势能之中, 可通过维里定理把体系的总能量化分为 热运动能量以及集体运动能量两部分 ${ }^{[18]}$. 具体计算 方法如下: 对每层中所有原子在 $z$ 方向上的速率加 和平均得到每层整体运动速率 $v_{\mathrm{c}}$, 进而获得铝膜整 体运动动能 $\mathrm{KE}_{\mathrm{c}}$. 将每个原子的 $z$ 方向速率减掉相应 $v_{\mathrm{c}}$ 之后可计算铝膜热运动动能 $\mathrm{KE}_{\mathrm{th}}$. 维里定理描述 原子热运动势能 $\mathrm{PE}_{\mathrm{th}}$ 等于热运动动能 $\mathrm{KE}_{\mathrm{th}}$, 可知 $E_{\mathrm{a}}^{\mathrm{th}}$ 等于 $2 \mathrm{KE}_{\mathrm{th}}$. 整体运动的势能 $\mathrm{PE}_{\mathrm{c}}$ 等于当前体系势能 $\mathrm{PE}_{\mathrm{sy}}$ 减掉热运动势能 $\mathrm{PE}_{\mathrm{th}},0 \textrm{K}$ 时体系的势能 $\mathrm{PE}_{0 \mathrm{~K}}$ 以 及热熔化潜热 $E_{\mathrm{m}}$. 最后 $E_{\mathrm{a}}^{\mathrm{c}}$ 通过 $\mathrm{PE}_{\mathrm{c}}$ 和 $\mathrm{KE}_{\mathrm{c}}$ 之和得到. 熔化潜热 $E_{\mathrm{m}}$ 通过将铝膜的液态原子数乘以铝材料 的单位熔化潜热而得到. 液态原子的判定方法是根 据局部序参数 (local order parameter) ${ }^{[25]}$, 该参数计算 方法见文献[18]的附录 $\mathrm{C}$ 和文献[25]. 单位熔化潜热 由计算铝材料在零压下液态与固态时的内能差获 得. 电子热能 $E_{\mathrm{e}}$ 为电子温度 $T_{\mathrm{e}}$, 电子热容 $C_{\mathrm{e}}$ 以及体积 等参数的乘积.

图 2 演示了脉宽为 $200 \mathrm{fs}$ 吸收强度为 $77 \mathrm{~J} \cdot \mathrm{m}^{-2}$ 的激光脉冲辐照前后铝膜的各种能量分布随时间 的变化. 由图 2 可以看出, 电子热能 $E_{\mathrm{e}}$ 在激光辐照后 快速上升, 在 $400 \mathrm{fs}$ 时达到约 $8000 \mathrm{eV}$. 电子热能 $E_{\mathrm{e}}$ 上升后, 原子热运动能 $E_{\mathrm{a}}^{\mathrm{th}}$ 随之上升. 约 $1.7 \mathrm{ps}$ 时 $E_{\mathrm{a}}^{\mathrm{th}}$ 达到其最高值 $15500 \mathrm{eV}$, 随后开始下降. 原子热运 动能量的增加来自通过能量转换的电子热能. 铝的 电子-声子耦合系数是 $4.9 \times 10^{17} \mathrm{~W} \cdot \mathrm{m}^{-3} \cdot \mathrm{K}^{-1}$, 高于镍 和金的(分别为 $3.6 \times 10^{17}$ 和 $2.1 \times 10^{16} \mathrm{~W} \cdot \mathrm{m}^{-3} \cdot \mathrm{K}^{-1}$ ), 因此 铝电子热能转变为原子热能的速率要比镍和金的 大. 约 $1.7 \mathrm{ps}$ 以后原子热能 $E_{\mathrm{a}}^{\mathrm{th}}$ 开始下降, 同时 $E_{\mathrm{a}}^{\mathrm{c}}$ 开 始以相似的速度上升. 因为晶格的快速升温会导致 铝膜形成压缩压力并引发铝膜的声子振动. 在振动
过程中原子热能和声子振动能量会相互转变, 形成 了它们之间互补的波动形状. 从图 2(a)中可以看出 原子热能和集体运动能的波动幅度越来越小, 表明 声子振动能逐步转变为原子的热能.

$E_{\mathrm{m}}$ 在约 $5 \mathrm{ps}$ 前的增加幅度很小, 表明铝膜的熔 化速度很慢. $5 \mathrm{ps}$ 以后熔化速度开始逐渐增大, 并在 $12 \mathrm{ps}$ 左右达到最大. $12 \mathrm{ps}$ 以后熔化速度又开始逐 渐减慢, 并在约 $14 \mathrm{ps}$ 时进入非常缓慢的熔化阶段.

图 3 表示出铝膜受飞秒激光辐照时其受辐照表 面和背面区域的电子和晶格温度差异. 从图 3 看出, 激光能量的吸收导致铝膜受辐照表面区域电子温 度 $T_{\mathrm{e}}^{\mathrm{ront}}$ 急剧上升, 以及背面区域的电子温度 $T_{\mathrm{e}}^{\mathrm{ear}}$ 几乎 同步地上升. 由于铝的电子热传导速度快, 因此在 约 $0.9 \mathrm{ps}$ 的时间内整个铝膜的电子温度达到平衡. 电子热能逐渐通过电子-声子耦合作用被转变为晶 格热能, 铝膜电子温度快速提高的同时, 晶格温度 也开始上升. 因为铝的电子-声子耦合强度以及电子 和晶格热传导速度较高, 因此镍和金分别需要 5 和 $15 \mathrm{ps}$ 才能把大部分电子热能转变为晶格热能 ${ }^{[9]}$, 但 铝仅需要 $1.5 \mathrm{ps}$ 即完成此过程, 并在 $5 \mathrm{ps}$ 时就达到 电子和晶格间的温度平衡.

从图 3 可以看出, 从 $2 \mathrm{ps}$ 开始辐照表面区域的 晶格温度 $T_{1}^{\text {front }}$ 开始低于背面区域的晶格温度 $T_{1}^{\text {rear }}$, 表 明表面区域先于背面区域熔化(对此图 4(a)将给出 更为清楚的显示).

在飞秒激光辐照下, 铝膜的晶格温度和内部压 力会发生剧烈变化. 考察这些变化对理解铝膜的熔 化动力学很有帮助. 图 4 描绘了铝膜受到脉宽为 $200 \mathrm{fs}$, 吸收强度为 $77 \mathrm{~J} \cdot \mathrm{m}^{-2}$ 的激光辐照后晶格温度 和压力随空间和时间的变化. 


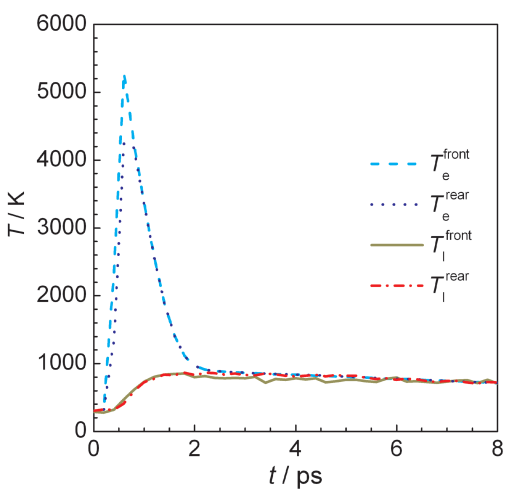

图 3 飞秒激光辐照铝膜后铝膜电子和晶格温度 随时间的变化

Fig.3 Time dependence of electron and lattice temperatures of the Al film irradiated with a femtosecond laser

Pulse width is $200 \mathrm{fs}$ and absorbed fluence is $77 \mathrm{~J} \cdot \mathrm{m}^{-2}$; $T_{\mathrm{e}}^{\text {front }}$ or $T_{1}^{\text {front }}$ : the electron or lattice temperature at the front surface of the film, $T_{\mathrm{e}}^{\text {rear }}$ or $T_{1}^{\text {rear }}$ : the electron or lattice temperature at the rear surface of the film

铝膜的表面和背面受辐照后由于热膨胀而向 外扩张并由此引起振动, 振动的振幅随时间逐步减 小. 图 4(a)中晶格温度的变化受诸多因素和过程的 影响. 首先, 能量从高温电子转移到晶格导致晶格 温度上升, 由于铝的电子-声子耦合强度较高以及电 子和晶格的热传导速率较快, 铝膜受辐照表面和背 面的电子温度以及晶格直接受辐照的部分和其他 部分温度差别不显著. 随着熔化的开始, 部分热能 会转换为熔化潜热导致晶格温度下降. 图 4(a)显示 铝膜快速熔化时晶格温度明显下降.

铝膜会以弹性振动的方式释放激光辐照产生 的压力. 这一过程对铝膜温度的变化也有显著影
响. 图 4(b) 显示飞秒激光辐照导致铝膜中央产生高 达 $3.8 \mathrm{GPa}$ 的压缩压力, 这种压缩压力的释放引起 铝膜中部张应力集中以致膨胀, 并最终引起铝膜两 边边界的扩张与收缩, 即铝膜的振动. 图 4(b)中振动 振幅在逐渐变小, 因此振动在逐步减弱. 图 2 中铝膜 集体运动能量峰值逐渐下降也说明这一点: 铝膜的 集体运动能在吸收总能量中所占比例从 $8.5 \mathrm{ps}$ 时的 $22.5 \%$ 下降到 $105 \mathrm{ps}$ 时的 $17.6 \%$.

电子-声子耦合强度直接影响铝膜不同深度位 置温度梯度的产生. 如果耦合强度较高, 电子在热 传导的同时, 在受激发区附近将大量能量传递给晶 格, 从而形成晶格的温度梯度. 镍的强电子-声子耦 合导致其在激光辐照后约 $10 \mathrm{ps}$ 内形成了明显的晶 格温度梯度 ${ }^{[18]}$. 相反, 对于金来说, 较弱的电子-声子 耦合以及较快的导带电子能量输运导致金属膜的 整体温度提升, 其结果发生均质熔化. 虽然铝的电 子-声子耦合强度较高, 但是它的电子热传导速度也 较快, 因此可以看到图 4(a)中没有形成明显的温度 梯度.

图 4(a,b)中的一对虚实线划分开了铝膜中每个 时刻的固态和液态区域. 从这对线的变化可以看 出, 整个铝膜的熔化分为两个步骤: 在辐照开始后 约 8-13 ps 时间内, 从受辐照表面开始铝膜的约 90\%区域快速地熔化; 紧接着是一个相对较慢的熔 化过程, 以至到 $100 \mathrm{ps}$ 时铝膜背面区域仍然有固态 原子存在. 铝膜表面区域在激光辐照后约 3 ps 即开 始熔化, 此时的温度约为 $800 \mathrm{~K}$, 明显高于铝在零压 下的熔化温度 $(650 \mathrm{~K})$. 该温度为应用 EAM 势以及 前述铝的相关参数值进行 MD模拟计算得到的铝在
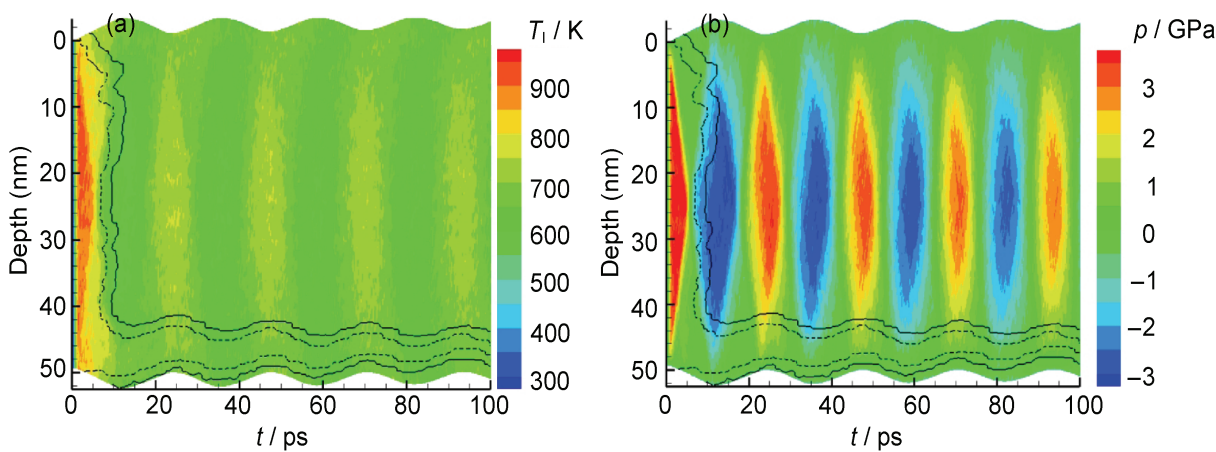

图4飞秒激光辐照铝膜后晶格温度、压力随空间和时间变化的等值线图

Fig.4 Contour plots of lattice temperature and pressure of the Al film irradiated with a femtosecond laser as functions of depth and time

Pulse width is $200 \mathrm{fs}$ and absorbed fluence is $77 \mathrm{~J} \cdot \mathrm{m}^{-2}$; solid and dashed lines show the beginning and the end of the melting process, defined by the first time when the fractions of the liquid atoms in a layer are achieved $10 \%$ and $90 \%$, respectively. 

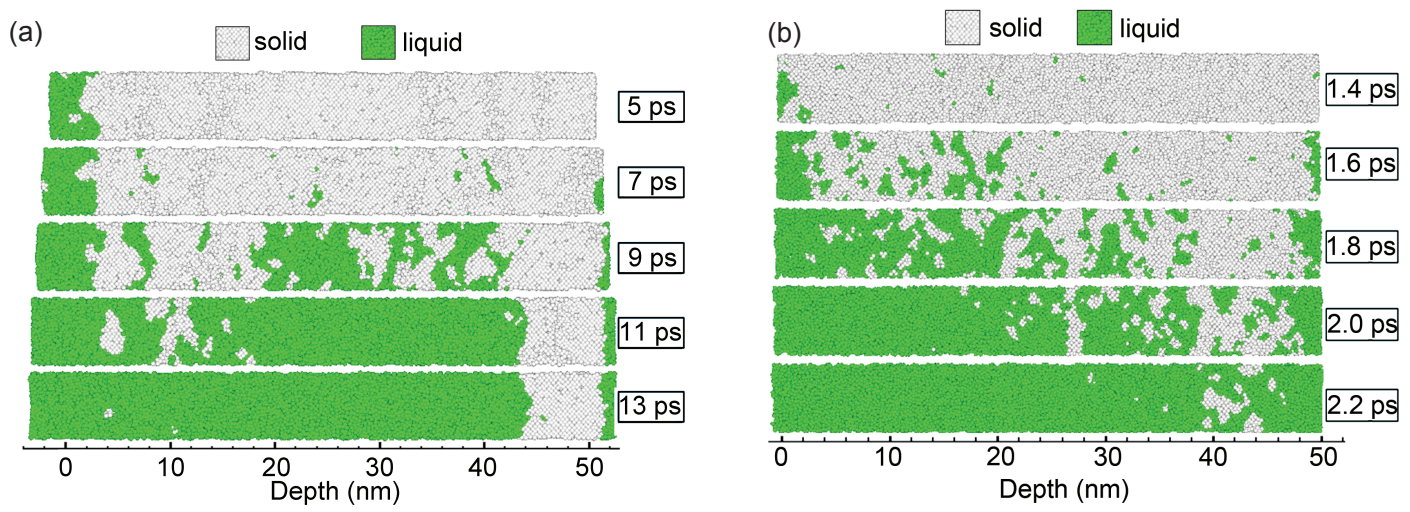

图 5 飞秒激光辐照铝膜后的熔化过程快照

Fig.5 Snapshots from simulation of laser melting of the Al film irradiated with a femtosecond laser Laser pulse width is $200 \mathrm{fs}$ and absorbed fluences are $77 \mathrm{~J} \cdot \mathrm{m}^{-2}$ (a) and $157 \mathrm{~J} \cdot \mathrm{m}^{-2}($ b).

零压下固液相共存时的平衡温度 ${ }^{[25]}$. 铝膜的大部分 区域熔化发生在其后约 $10 \mathrm{ps}$ 内, 随后仅剩下背面附 近有固态原子存在. 同时还可从图 4(b)观察到, 背面 附近区域原子发生了非均质熔化, 即熔化前沿缓慢 向中央推进.

为了详细观察快速熔化现象, 图 5(a) 显示出了 在 5-13 ps 时间段内 5个原子结构的快照图. 图中根 据局部序参数对原子进行了染色: 两种颜色分别代 表原子处在局部固相和液相环境中. 为了减少原子 热振荡的影响, 快照中原子的位置按照每 100 个 $\mathrm{MD}$ 积分步中各原子位置的平均值来计算的. 这些 快照显示铝膜的熔化是一个复杂的均质熔化过程: 在熔化的前方并不全是固态晶体, 而是生成了许多 小的液态区域. 这些微小的液态区域随时间推移逐 渐长大、合并, 使熔化前沿得以高速传播, 达到约 $10000 \mathrm{~m} \cdot \mathrm{s}^{-1}$.

从图 4(a)可以看出, 在铝膜辐照表面附近区域 初始熔化时的温度约为 $800 \mathrm{~K}$, 而在铝膜中部初始 熔化时的温度最低只有约 $640 \mathrm{~K}$, 略低于铝在零压 下熔化温度 $(650 \mathrm{~K})$. 这一现象来自如下事实: 在这 些区域的熔化发生时, 相应压力值为负. 铝膜背面 附近部分区域出现的是相对较慢的非均质熔化, 但 没有发现前面讨论过的快速均质熔化. 图 4(b) 则显 示出铝膜受辐照背面附近的固态区分别邻接着快 速均质熔化形成的液态区和铝膜背面开始的非均 质熔化液态区.

更高强度的激光会使整个铝膜发生更快的均 质熔化. 图 5(b)为在脉宽 $200 \mathrm{fs}$, 吸收强度为 $157 \mathrm{~J}$. $\mathrm{m}^{-2}$ 的激光辐照下铝膜熔化的快照图. 可以看出, 铝 膜表面在激光辐照约 1.4 ps 后即开始熔化; 在约 1.6 ps 时大部分区域已经变成液态成核区; 在约 $2.2 \mathrm{ps}$ 时绝大部分铝膜熔化完毕; 这一熔化过程只需大约 $0.8 \mathrm{ps}$ 时间(1.4-2.2 ps). 这个模拟结果与 Kandyla 等 ${ }^{[8]}$ 的实验结论一致, 他们在类似的激光强度范围 内得到铝膜的熔化时间为 1.5-2 ps.

从图 5(b)中的快照看出, 与较低的吸收强度(77 $\left.\mathrm{J} \cdot \mathrm{m}^{-2}\right)$ 下的熔化过程不同, 较高吸收强度下均质熔 化过程中产生了更多的液态成核区域. 这些液态成 核区域以更快的速度长大和结合, 只需用长约 0.8 ps 的时间就能完成绝大部分区域的熔化. 这一时间 小于金的超快晶体结构崩塌时间 $(2 \mathrm{ps})^{[18]}$, 只有不到 10 个原子振动的周期. 由于铝膜晶体结构的温度超 过了晶体稳定的极限高温, 因而在辐照后约 $1.4 \mathrm{ps}$ 时刻开始了超快的晶体结构崩塌.

\section{3 结 论}

通过耦合的 TTM-MD 模拟计算方法研究了飞 秒激光辐照下厚度为 $49.3 \mathrm{~nm}$ 铝膜的温度、压力变 化情况和熔化机制.

结果表明受到超短激光辐射后铝膜的温度和 压力会经历较复杂的变化, 这些变化影响铝膜的熔 化机理. 铝膜晶格温度快速升高且整体一致, 这一 特点导致铝膜的熔化与镍、金薄膜的熔化不一致. 在激光强度较高时, 铝膜熔化得比镍和金都更快, 且铝膜是全局一致的熔化而镍膜在受辐照方向的 一半熔化早于另一半; 金膜虽然也是发生全局一致 的熔化, 但相对铝膜要慢得多.

和金属镍、金相似, 铝膜存在三个清晰的激光 诱导熔化机制: 在晶体内部夜态成核并长大的均质 熔化, 固液界面从铝膜受激光辐照背面液态区域均 
质熔化形成的液态区向晶体内部逐步推进的非均 质熔化, 以及因为晶体结构的温度超过了晶体稳定 的极限高温从而在短期内发生的超快晶体结构崩 塌. 模拟观察到的超快晶体结构崩塌熔化时间与实 验测量的超强激光诱导的铝膜熔化时间一致.

致谢：感谢 ZHIGILEI Leonid V. (Department of Materials Science and Engineering, University of Virginia, USA)提供的 计算程序和许多有益的讨论.

\section{References}

1 Tabata, N.; Yagi, S.; Hishii, M. J. Mater. Process. Technol., 1996, 62: 309

2 Yeo, C. Y.; Tam, S. C.; Jana, S.; Lau, M. W. J. Mater. Process. Technol., 1994, 42: 15

3 Sun, Z.; Ion, J. C. J. Mater. Sci., 1995, 30: 4205

4 Gu, B. Proceedings of the Society of Photo-Optical Instrumentation Engineers (SPIE), 2006, 61: 10601

5 Wang, C. W.; Qu, S. Y.; Wang, X. H.; Sun. J. H. Machinist Metal Forming, 2008, 8: 38 [王承伟, 曲仕尧, 王新洪, 孙俊华. 金属 加工: 热加工, 2008, 8: 38]

6 Cao, X.; Jahazi, M.; Immarigeon, J. P.; Wallace, W. J. Mater. Process. Technol., 2006, 171: 188

7 Guo, C.; Rodriguez, G.; Lobad, A.; Taylor, A. J. Phys. Rev. Lett., 2000, 84: 4493

8 Kandyla, M.; Shih, T.; Mazur. E. Phys. Rev. B, 2007, 75: 214107

9 Siwick, B. J.; Dwyer, J. R.; Jordan, R. E.; Miller, R. J. D. Science, 2003, 302: 1382

10 Dwyer, J. R.; Jordan, R. E.; Hebeisen, C. T.; Harb, M.; Ernstorfer,
R.; Dartigalongue, T.; Miller, R. J. D. J. Mod. Opt., 2007, 54: 905

11 Rahman, A. Phys. Rev. A, 1964, 136: 405

12 Stillinger, F. H.; Rahman, A. J. Chem. Phys., 1974, 60: 1545

13 Hou, H. Y.; Chen, G. L.; Chen, G. Acta Phys. -Chim. Sin., 2006, 22: 771 [侯怀宇, 陈国良, 陈 光. 物理化学学报, 2006, 22: 771]

14 Zhang, T.; Zhang, X. R.; Wu, A. L.; Guan, L.; Xu, C. Y. Acta Phys. -Chim. Sin., 2003, 19: 709 [张＼cjkstart㢷, 张晓茹, 吴爱玲, 管 立, 徐昌业. 物理化学学报, 2003, 19: 709]

15 Zhou, G. R.; Li, B. Q.; Geng, H. R.; Teng, X. Y.; Chen, G. L. Acta Phys. -Chim. Sin., 2007, 23: 1071 [周国荣, 李蓓琪, 耿浩然, 腾新营, 陈广利. 物理化学学报, 2007, 23: 1071]

16 Xin, J. T.; Zhu, W. J.; Liu, C. L. Explosion and Shock Waves, 2004, 24: 207 [辛建婷, 祝文军, 刘仓理. 爆炸与冲击, 2004, 24: 207]

17 Anisimov, S. I.; Kapeliovich, B. L.; Perel'man, T. L.; Eksp, Z. Soviet Physics-JETP, 1974, 39: 375

18 Ivanov, D. S.; Zhigilei, L. V. Phys. Rev. B, 2003, 68: 22

19 Schäfer, C.; Urbassek, H. M.; Zhigilei, L. V. Phys. Rev. B, 2002, 66: 4

20 Daw, M. S.; Baskes, M. I. Phys. Rev. Lett., 1983, 50: 1285

21 Daw, M. S.; Baskes, M. I. Phys. Rev. B, 1984, 29: 6443

22 Zhou, X. W.; Wadley, H. N. G.; Johnson, R. A.; Larson, D. J.; Tabat, N.; Cerezo, A.; Petford-Long, A. K.; Smith, G. D. W.; Clifton, P. H.; Martens, R. L.; Kelly, T. F. Acta Mater, 2001, 49: 4005

23 Volkov, A. N.; Zhigilei, L. V. Journal of Physics Conference Series, 2007, 59: 640

24 Tas, G.; Maris, H. J. Phys. Rev. B, 1994, 49: 15046

25 Morris, J. R.; Song, X. J. Chem. Phys., 2002, 116: 9352 